\title{
Treatment of Patients in Coma and Exercises for Enhancing Cognitive Functions after Traumatic Brain Injury
}

Theofilidis Antonis

Clinical Neuropsychologist University of Western Makedonia- Greece Occupational Therapy Department

Corresponding Author: Theofilidis Antonis, Clinical Neuropsychologist Githiou 1, Thessaloniki, Greece. Received date: July 15, 2021; Accepted date: Augusł 03, 2021; Published date: August 13, 2021

Citation: Theofilidis Antonis. (2021) Treatment of Patients in Coma and Exercises for Enhancing Cognitive Functions after Traumatic Brain Injury. J. Neuroscience and Neurological Surgery. 9(4); DOI:10.31579/2578-8868/193

Copyright: (C) 2021 Theofilidis Antonis, This is an open-access article distributed under the terms of The Creative Commons Attribution License, which permits unrestricted use, distribution, and reproduction in any medium, provided the original author and source are credited

\begin{abstract}
The neuropsychological training methods for the restoration of sensory losses after a TBI aim both at the restoration of the sensory losses and at the practice of the other senses to "compensate" for the dysfunctional sensation.

Aim: The purpose of the study was to present treatment of patients in coma and exercises for enhancing cognitive functions after traumatic brain injury.

Results: Electronically assisted treatment methods (Computer - Assisted Treatment, CAT) are now recognized methods, with high success rates in the rehabilitation of patients after TBI.

Conclusions: Cognitive rehabilitation must be done taking into account a variety of neurofunctional abilities and weaknesses. Basic skills need to be stimulated before any approach to more complex ones can be made. The use of audiovisual media in general contributes both to the restoration of vision and compensatory restoration of functions as well as to the general restoration of the patient's cognitive functions. Cognitive rehabilitation to recover and improve attention and memory, offers a variety of different types of exercises for specific disorders. Exercise can vary in degree of difficulty depending on the patient's needs.
\end{abstract}

Key-words: migraine; tanacetum parthenium; salix alba

\section{Introduction}

Acquired traumatic brain injury (TBI) is the leading cause of death and disability in young adults. It is estimated that 180,000 deaths from injuries are reported annually in developed countries, of which approximately $60 \%$ are due to brain injury. In addition, approximately 200,000 people acquire a permanent disability (prevalence) after a TBI. Also, about 2 million people are examined annually as outpatients, of whom $1 / 4$ end up being treated with some degree of TBI. From the above indicators it is understood that trauma and primarily brain trauma is a serious public health problem worldwide.Of the patients treated, approximately $80 \%$ have mild traumatic injury (Glasgow Scale score 14-15) and require little or no care in an intensive care unit (ICU). About $10 \%$ have moderate traumatic brain injury (Glasgow Scale 9-13) and may need ICU admission for simple observation. For the remaining $10 \%$, who present with severe traumatic brain injury (Glasgow Scale 3-8), rapid surgery and stabilization in the ICU play an important role in improving patient morbidity and mortality (Rossi \& Castioni, 2008).

It has been proven that the largest percentage of TBI occurs at the time of injury and in most cases is irreversible (primary damage). Taking precautionary measures (use of protective equipment, suitable clothing, etc.) may reduce the rate of initial damage. Recent studies demonstrate the existence of additional progressive brain damage for several hours and days after injury. Secondary symptoms (such as increased endocrine hypotension or episodes of hypotension and hypoxia), dramatically aggravate the course of TBI and significantly affect the final outcome of patients. The treatment approach of physicians in trauma centers (interdisciplinary treatment team) is oriented towards the prevention or treatment of clinical findings that may aggravate the patient's condition with this additional or "secondary brain damage" (Bigler, 2016). TBI, regardless of the degree of severity (mild, moderate, severe), bring about a change in the patient's ability to communicate and react.

\section{Treatment of patients in coma}

Regardless of the severity of traumatic brain injury, they also cause a change in the individual's ability to interact with the external environment, a phenomenon also known as a change in the patient's level of consciousness. drowsiness and lethargy, as the reduction of reactions and reaches a coma (Gosseries et al., 2011).

Coma is the complete atony of consciousness and the inability to respond to environmental stimuli, except in a reflexive way. Both the degree and duration of the coma reflect the severity of the brain injury. The longer the patient remains in a coma, the less likely he or she is to develop well. Only after adequate vigilance is restored can the characters and properties of the patient's reactions be assessed (Gosseries et al., 2011).The importance of the patient's reactions is the most important element that is evaluated in determining any alterations in the patient's behavior, personality, and quality of life. Changes due to TBI range from mild to very significant and are difficult to overlook due to the complexity of the brain (Gosseries et al., 2011).

\section{Plant condition}

Prolonged vegetative state is sometimes a consequence of coma. The patient lacks the abilities of autonomous and spontaneous thinking and 
perception of their environment, but nevertheless retains his functional abilities and the normal type of sleep. The brain has limited function, the patient's superior cognitive and spiritual abilities are lost, but without affecting to a large extent its vital functional systems (respiratory and circulatory).Patients with long-term vegetative state may have limited reflex movements, such as the ocular response to external stimuli or expressions of sadness and joy on the face, yet they are unable to communicate verbally and respond to commands. Depending on the cause of the coma, special treatment is given to treat the coma, which should be started as soon as possible to avoid further impairment of the patient's abilities. During coma or later in the vegetative state the therapeutic approaches focus on the aesthetic stimulation of the patient and the training of relatives in ways of communication and interaction with the patient. As patient stimulation improves, therapeutic approaches focus on maintaining attention and performing key activities, reducing confusion, and orienting the individual.

\section{The use of videos - Recordings in treatment}

The neuropsychological training methods for the restoration of sensory losses after a TBI aim both at the restoration of the sensory losses and at the practice of the other senses to "compensate" for the dysfunctional sensation. Existing methods can be divided into three groups: Visual Scanning Training (VST), Audio-Visual Scanning Training (AViST) and Vision Restoration Training (VRT). VST and AViST techniques aim to compensate for vision loss by training eye scan movements (or practicing the auditory nerve in the case of AViST), while VRT techniques aim to improve visual impairments by activating residual visual functions by the use of light and the achievement of visual stimuli (Dundon, Bertini, Làdavas, Sabel \& Gall, 2015).

The use of audiovisual media in general contributes both to the restoration of vision and compensatory restoration of functions as well as to the general restoration of the patient's cognitive functions (Dundon, Bertini, Làdavas, Sabel \& Gall, 2015).

\section{The use of computers in reconstruction}

Electronically assisted treatment methods (Computer - Assisted Treatment, CAT) are now recognized methods, with high success rates in the rehabilitation of patients after TBI.

CAT programs are available as software or applications for mobile and PC. These programs usually consist of repetitive tests aimed at specific skills or functional tasks that are tailored to the individual's performance. This ensures that "the speed and complexity of the exercises increase the user's performance and are constantly improved in order to maintain a consistently high percentage of successful trials while stimulating the patient's brain with gradually more demanding tasks" (Lebowitz, DamsO'Connor \& Cantor, 2012).

This technology is used as an adjunct to specialized clinical therapies to treat certain areas of cognitive and communication deficits, providing consistent feedback to the individual (Lebowitz, Dams-O'Connor \& Cantor, 2012).

\section{Exercises for enhancing cognitive functions after TBI}

Cognitive rehabilitation must be done taking into account a variety of neurofunctional abilities and weaknesses. Basic skills need to be stimulated before any approach to more complex ones can be made, ie perception, concentration and attention are restored or even worked on before complex mental functions. Brain rehabilitation goes hand in hand with brain development (Theofilidis, et. al, 2020).

\section{Perception}

The types of aesthetics that are disturbed in the cases of patients with perceptual disorders are vision (homonymous semianopsis), hearing, touch, temperature, pain, sensation of vibration and susceptibility. The patient tends to perceive all stimuli as pain. He despises the suffering side, has a disturbance in the perception of his body image and a reduced perception of time and space (Brubaker, 2008).

Perception exercises have different areas depending on the sensation that has been most damaged as a result of TBI. The practice of visual perception, for example, is usually achieved through the use of various exercises such as paper and pencil, the use of different colors or a variety of light intensity, etc.

Exercises focus on understanding the visual stimuli, recognizing and responding to the patient. Respectively, with the help of other means / multimedia or techniques, the other sensory deficiencies are practiced with the ultimate goal of strengthening the patients' perception (Brubaker, 2008).

\section{Attention and memory}

Cognitive rehabilitation to recover and improve attention and memory, offers a variety of different types of exercises for specific disorders. Exercise can vary in degree of difficulty depending on the patient's needs (Powell \& Malia, 2008).

Difficulties related to selective attention, the ability to concentrate on important things while ignoring insignificant stimuli. Exercises are analogous to those of improving general attention, only adding an external stimulus such as noise or music (Powell \& Malia, 2008).

Many patients experience distractions due to various thoughts and anxieties. In this case the patient is encouraged to write his thoughts on a piece of paper before starting the procedure he wants to perform or even discussing them with the therapist for psychotherapy (Powell \& Malia, 2008).

Deficiencies of the type associated with shared attention when attention needs to be shared between two things or situations at the same time. A good practice for improving this type of defective attention requires listing the odd or even numbers until the therapist triggers the change and the patient begins counting the other category of numbers. Exercise can be modified by listing other numbers or words (Powell \& Malia, 2008). Distinctive attention disorders require similar exercises. For this type of disorder, practice involves doing two tasks at the same time (Powell \& Malia, 2008).

Memory is also directly dependent on various other functions that may have been damaged, such as attention. Since the human mind is programmed to be able to process only things and situations to which attention has been paid, the patient with deficient attention cannot process any information. In these cases the patient can improve it his memory by performing mnemonic exercises through categorization, ie making some lists of the necessary movements or objects or even actions that he must perform in order to achieve his goal (Powell \& Malia, 2008).

\section{Language and thought - Problem solving}

Exercises for recovering and improving defective problem solving are based on abstract ability and thinking. Cognitive interventions in this category work equally well at either the individual or group therapy level (Theofilidis, et. al, 2020). In the second case, the goal is not only to train the patient but also to socialize the patients. Therapeutic groups are strictly limited to 4 people (Brubaker, 2008).

Interventions of this kind are divided into 3 distinct stages which are the following:

Intervention stage 1 (educational basis). The goal of this stage is to understand the mechanisms, effects of injury, changes, emotional and behavioral changes as well as the role of anesthesia (Brubaker, 2008).

Intervention stage 2 (practical basis). At this stage, the aim is to address the changes and to define an effective strategy for the improvement of the shortcomings and the solution of the problems (Brubaker, 2008).

Stage 3 intervention (internship). In this last stage, the treatment team helps and guides the patient to perform a variety of exercises of attention, memory, executive function, visual processing. 


\section{Conclusions}

Cognitive rehabilitation must be done taking into account a variety of neurofunctional abilities and weaknesses. Basic skills need to be stimulated before any approach to more complex ones can be made. The use of audiovisual media in general contributes both to the restoration of vision and compensatory restoration of functions as well as to the general restoration of the patient's cognitive functions. Cognitive rehabilitation to recover and improve attention and memory, offers a variety of different types of exercises for specific disorders. Exercise can vary in degree of difficulty depending on the patient's needs.

\section{References}

1. Bigler, E.D. (2016). Systems Biology, Neuroimaging, Neuropsychology, Neuroconnectivity and Traumatic Brain Injury. Frontiers in Systems Neuroscience, 10, 55.

2. Brubaker, S.H. (2008) Workbook for Cognitive Skills: Exercises for Thought-processing and Word Retrieval. ( $2^{\text {nd }}$ Edition) p. 1525 Wayne State -University Press.

3. Dundon, N.M., Bertini, C., Làdavas, E., Sabel, B.A. \& Gall, C. (2015). Visual rehabilitation: visual scanning, multisensory stimulation and vision restoration trainings. Front. Behav. Neurosci. 9:192. doi: 10.3389/fnbeh.2015.0019
4. Gosseries, O., Vanhaudenhuyse, A., Bruno, M.A., Demertzi, A., Schnakers, C., Boly, M., Maudoux, A., Moonen, G. \& Laureys, S. (2011). Disordersof consciousness: coma, vegetative and minimally consciousstates. In: Cvetkovic D, Cosic I (eds) States of consciousness, thefrontiers collection. Springer, Heidelberg, pp 29-55.

5. Lebowitz, M.S., Dams-O'Connor, K. \& Cantor, J.B. (2012). Feasibility of computerized brain plasticity-based cognitive training after traumatic brain injury. Journal of Rehabilitation Research and Development, 49(10), 1547-1556.

6. Powell, T. \& Malia, K. (2008). Brain Injury Workbook: Exercises for Cognitive Rehabilitation. Journal of Occupational Psychology, Employment and Disability, Vol 10, No 2, Autumn 2008.

7. Theofilidis Antonis, Sofologi Maria, Fountoulakis Kostas, Nimatoudis John. «Interesting Case of Traumatic Brain Injury Neuropsychological Assessmen». Case Report. Clinical Cases in Medicine.International, Peer Reviewed, Open Access Journal.2020;1(1):CCM-01-1001- Apr 10, 2020-MedText Publications. $\mathrm{http} / / / \mathrm{www} . \mathrm{medtextpublications.com/clinical-cases-in-medicine-}$ home.php
This work is licensed under Creative

Commons Attribution 4.0 License

To Submit Your Article Click Here: Submit Manuscript

DOI: $10.31579 / 2578-8868 / 193$

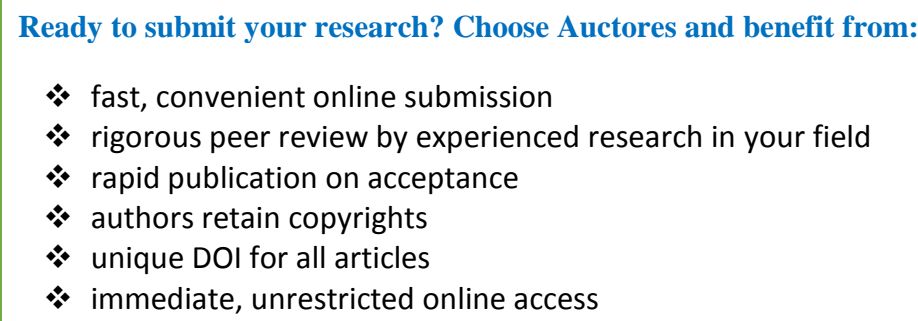

Ready to submit your research? Choose Auctores and benefit from:

* fast, convenient online submission

* rigorous peer review by experienced research in your field

* rapid publication on acceptance

* authors retain copyrights

* unique DOI for all articles

* immediate, unrestricted online access

At Auctores, research is always in progress.

Learn more www.auctoresonline.org/journals/neuroscience-andneurological-surgery 